\title{
Effective collecting area of a cylindrical Langmuir probe in magnetized plasma
}

Mariia Usoltceva, Eric Faudot, Stéphane Devaux, Stéphane Heuraux, Jordan Ledig, Georgiy V. Zadvitskiy, Roman Ochoukov, Kristel Crombé, and Jean-Marie Noterdaeme

Citation: Physics of Plasmas 25, 063518 (2018); doi: 10.1063/1.5028267

View online: https://doi.org/10.1063/1.5028267

View Table of Contents: http://aip.scitation.org/toc/php/25/6

Published by the American Institute of Physics

\section{Articles you may be interested in}

A detailed study on the structures of steady-state collisionless kinetic sheath near a dielectric wall with secondary electron emission. I. Classic sheath and its structure transition

Physics of Plasmas 25, 063519 (2018); 10.1063/1.5023794

A detailed study on the structures of steady-state collisionless kinetic sheath near a dielectric wall with secondary electron emission. II. Inverse and space-charge limited sheaths

Physics of Plasmas 25, 063520 (2018); 10.1063/1.5025137

Electron collection and thermionic emission from a spherical dust grain in the space-charge limited regime Physics of Plasmas 25, 063701 (2018); 10.1063/1.5032153

Electron reflection effects on particle and heat fluxes to positively charged dust subject to strong electron emission

Physics of Plasmas 25, 063702 (2018); 10.1063/1.5026384

Emitting large dust grains: Floating potential and potential wells

Physics of Plasmas 25, 063703 (2018); 10.1063/1.5010042

Extraction of nonlinear waveform in turbulent plasma

Physics of Plasmas 25, 062304 (2018); 10.1063/1.5027124

\section{PHYSICS TODAY}

MANAGER'S GUIDE

WHITEPAPERS

\section{READ NOW}

PRESENTED BY

Accelerate R\&D with Multiphysics Simulation
○OMSOL 


\title{
Effective collecting area of a cylindrical Langmuir probe in magnetized plasma
}

\author{
Mariia Usoltceva, ${ }^{1,2,3, a)}$ Eric Faudot, ${ }^{2}$ Stéphane Devaux, ${ }^{2}$ Stéphane Heuraux, ${ }^{2}$ \\ Jordan Ledig, ${ }^{2}$ Georgiy V. Zadvitskiy, ${ }^{2,4,5}$ Roman Ochoukov, ${ }^{3}$ Kristel Crombé, ${ }^{1,6}$ \\ and Jean-Marie Noterdaeme $e^{1,3}$ \\ ${ }^{1}$ Ghent University, 9000 Gent, Belgium \\ ${ }^{2}$ Université de Lorraine, 54506 Vandouvre-lès-Nancy, France \\ ${ }^{3}$ Max-Planck-Institut für Plasmaphysik, Boltzmannstr. 2, 85748 Garching, Germany \\ ${ }^{4}$ Institut für Grenzflächenverfahrenstechnik und Plasmatechnologie, Universität Stuttgart, 70569 Stuttgart, \\ Germany \\ ${ }^{5}$ CEA, IRFM, F-13108 Saint-Paul-lez-Durance, France \\ ${ }^{6}$ LPP-ERM/KMS, Royal Military Academy, 1000 Brussels, Belgium
}

(Received 8 March 2018; accepted 18 May 2018; published online 14 June 2018)

\begin{abstract}
Langmuir probe diagnostic on magnetic plasma devices often encounters more challenges in data processing than in non-magnetized plasmas, the latest itself being far from simple. In this paper, a theory of particle collection by a probe at the plasma potential in collisionless weakly ionized plasmas is constructed, accounting for velocities distributed according to the Maxwell equation and different mechanisms of particle collection depending on their speed. Experimental validation of the presented theory has been done with 2 cylindrical probes $\left(\mathrm{r}_{\mathrm{pr}}=75 \mu \mathrm{m}\right.$ and $\mathrm{L}_{\mathrm{pr}}=1 \mathrm{~cm}$ and $\mathrm{r}_{\mathrm{pr}}$ $=0.5 \mathrm{~mm}$ and $\mathrm{L}_{\mathrm{pr}}=1 \mathrm{~cm}$ ) parallel to $\overrightarrow{\mathrm{B}}$ on a linear plasma device Aline, with magnetic fields of $0.0024-0.1 \mathrm{~T}$ and plasma densities of $10^{15}-10^{17} \mathrm{~m}^{-3}$ in helium. Cylindrical probe measurements are compared to data from a planar probe perpendicular to the magnetic field, and the results for electron density, temperature, and plasma potential are presented. The introduced theory is initially constructed for a cylindrical probe but is applicable to various probe sizes, shapes, and orientations. Alongside the main subject, a number of associated issues are addressed with different details: a probe design issue relative to the magnetized environment, the "intersection" method of plasma potential evaluation, and the robustness of the conventional "1st derivative" method, a current bump near the plasma potential, lower limit for electron temperature estimation, and self-consistent calculation of electron temperature and density. https://doi.org/10.1063/1.5028267
\end{abstract}

\section{INTRODUCTION}

A complete theory of Langmuir probes in a magnetic field has never been developed. Plasma collisionality and the magnetization level, as well as the probe type and orientation, each affects significantly the approach of data interpretation. An extensive list of references for various probes in various conditions can be found in Ref. 1 .

Of those works, very few tried to examine cylindrical probes. $^{2-8}$ Two articles published by Laframboise and Rubinstein ${ }^{2,3}$ can be considered as foundational theoretical papers on the subject of a cylindrical Langmuir probe in magnetized plasmas. We will make an attempt to compare our model to theirs.

In this work, we focus only on the characterization of the electron current collected by a probe at the plasma potential $\left(\mathrm{V}_{\mathrm{pl}}\right)$ where no sheath is present, as it is the simplest technique for deriving the electron density. It allows us to avoid additional errors in particle motion calculation caused by the presence of a sheath around the probe. For simplicity, the ion collection at $\mathrm{V}_{\mathrm{pl}}$ is considered to be negligible.

In magnetized plasmas, less current is collected by a cylindrical Langmuir probe. It has been first shown in the

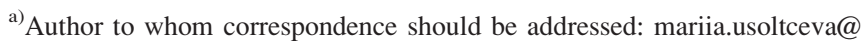
ipp.mpg.de
}

theoretical papers of Laframboise and Rubinstein ${ }^{2,3}$ and then observed in experiments. ${ }^{4} \mathrm{~A}$ widely used procedure in the presence of a magnetic field is to regard charged particle trajectories as helices around magnetic field lines that intersect only the area of the probe perpendicular to the magnetic field (or its projection on the perpendicular plane). This theory works well when the characteristic size of the probe in the perpendicular plane $d_{\perp}$ is much bigger than the Larmor radius $r_{c}$ and than the probe size in the parallel direction $d_{\|}$

$$
d_{\perp} \gg r_{c}, d_{\perp} \gg d_{\|}
$$

Since the ion Larmor radius is typically at least one order of magnitude larger than the electron one, these conditions in weak and medium magnetic fields are fulfilled only for electrons, while the ions rotate on the orbits significantly bigger than a probe size. Then, the common approach is to treat ions as unmagnetized. ${ }^{4}$

In this work, we make an attempt to fill in the gap in the theory for the conditions when (1) is not true, i.e., the probe parallel size is larger or comparable to the perpendicular one and the Larmor radius is not limited, i.e., for any magnetic field. This is the case when a cylindrical Langmuir probe is inserted in a plasma parallel to the magnetic field lines or at a small angle. The perpendicular projection of the probe surface is very small compared to the whole probe surface in 
this case and is obviously not applicable as a correct collecting surface. A substantial fraction of the current is collected by the probe side surface, and it must be taken into account. Otherwise, an underestimated collecting area would lead to an overestimated density value.

This paper presents a theory of the "effective" collecting area of a probe biased to $\mathrm{V}_{\mathrm{pl}}$. The theory accounts for particle velocities distributed according to the Maxwell equation and different mechanisms of particle collection depending on their speed. We assume the absence of any additional perpendicular drifts, diffusion, or anomalous transport in plasmas. Initially constructed for the conditions when (1) is not true, the theory is not limited to those, suggesting a solution for any ratio of the Larmor radii to probe sizes by the means of converging to the conventional unmagnetized theory when particle orbits are significantly bigger than a probe characteristic size. The theory is presented for electrons but might be useful for ions as well, when their contribution is significant.

The theory was validated on an experimental device which is described in Sec. II. In Sec. III, the theory is explained, its experimental application is presented, and a comparison to the Laframboise-Rubinstein theory is provided.

After the main subject of this paper-effective collecting area and density evaluation-some other issues are addressed which are typically bound to the IV curve analysis. In Sec. IV, we discuss different approaches of finding $\mathrm{V}_{\mathrm{pl}}$ and explain why the method of the intersection of linear fits of the transit and electron saturation regions is chosen. The robustness of the methods is compared.

A bump in the plasma current near $\mathrm{V}_{\mathrm{pl}}$ is observed in experiments. Its influence on the evaluation of correct $\mathrm{V}_{\mathrm{pl}}$ and current at $\mathrm{V}_{\mathrm{pl}}$ is also discussed in Sec. IV.

The electron temperature evaluation is done from a part of the region between the floating and plasma potentials, self-consistently with the density. The detailed procedure of accurate calculations is given in Sec. V.

\section{EXPERIMENTAL DEVICE}

The theory explained in this paper has been applied to process data on an experimental device. Aline (A LINear Experiment $)^{9,10}$ is a linear plasma chamber of $1 \mathrm{~m}$ length and $15 \mathrm{~cm}$ in radius. A capacitive discharge is created by a radio-frequency (RF) antenna operating at $25 \mathrm{MHz}$. The magnetic field can reach $0.104 \mathrm{~T}$. The neutral gas pressure was kept constant at $1 \mathrm{~Pa}$ for all presented results, and the gas used was helium.

An RF-compensated Langmuir probe ${ }^{11,12}$ with two exchangeable tungsten cylindrical tips of $\mathrm{r}_{\mathrm{pr}}=75 \mu \mathrm{m}$ and $\mathrm{L}_{\mathrm{pr}}=1 \mathrm{~cm}$ and $\mathrm{r}_{\mathrm{pr}}=0.5 \mathrm{~mm}$ and $\mathrm{L}_{\mathrm{pr}}=1 \mathrm{~cm}$ is installed on $\mathrm{a}$ 3D-movable manipulator parallel to the magnetic field direction. Each measurement is averaged over 20 sweeps of voltage from -70 to $70 \mathrm{~V}$, much longer than one RF period.

Measurements presented in this paper have been done at one spatial probe position above the RF cathode, $x=0 \mathrm{~mm}$, $\mathrm{y}=46 \mathrm{~mm}$, and $\mathrm{z}=-60 \mathrm{~mm}$ from the cathode center (which is $40 \mathrm{~mm}$ in radius), far enough to avoid the effects of strong RF potential distortion but at the same time close enough to the high density plasma region. The probe tip and the antenna are depicted in Fig. 1.

Perfect probe alignment is hardly possible in the experimental setup. To take this into account, we assume that the probe tip can deviate up to $5^{\circ}$ from the direction parallel to the magnetic field.

As a reference for density and temperature measurements, we use a planar probe perpendicular to the magnetic field $\left(r_{\mathrm{pr}}\right.$ $=0.5 \mathrm{~cm}$ ). The probe dimensions satisfy (1) for all values of the magnetic field used in our experiments. The theory for such a probe in a magnetic field is much simpler, and conventional methods are applicable for density extraction at $\mathrm{V}_{\mathrm{pl}}$.

We want to mention here that the density profiles provided in this paper should not be regarded as an attempt to give a dependency on the magnetic field. The coupled power was not constant at different B because of the difference in the matching quality. The RF antenna was directly connected to an RF amplifier (direct coupling), and measurements of the forwarded and reflected power were done. The difference between those, i.e., the coupled power, was varying nonlinearly between 5 and $35 \mathrm{~W}$.

Solely for the simplicity of representation, the data in all plots are grouped into 3 curves for 3 values of the forwarded power $(20,32$, and $51 \mathrm{~W})$. The presence of a probe did not affect the matching significantly, and so, we assume that the measurements were done in identical conditions with both cylindrical probes and the planar one.

\section{A. Criterion of plasma magnetization}

In weakly ionized plasmas, collisions with neutrals dominate over other sorts of collisions (electron-electron, ion-ion, and ion-electron). The cross section of inelastic electron-neutral collision is negligible for electron temperature $\mathrm{T}_{\mathrm{e}}<20 \mathrm{eV}$. $^{13}$ For elastic collisions, measurement results of cross sections are provided in Ref. 13 at different electron energies in Ar and He. For considered Aline conditions ( $5 \mathrm{eV}$ of $\mathrm{T}_{\mathrm{e}}$, gas $\mathrm{He}$ ), $\sigma_{\mathrm{e}-\mathrm{n}}=6$ $\times 10^{-20} \mathrm{~m}^{-2}$. The neutral density, calculated from $n=p / k T$ for $1 \mathrm{~Pa}$ and $300 \mathrm{~K}$, is equal to $n_{n}=2.4 \times 10^{20} \mathrm{~m}^{-3}$.

The mean free path of electrons is

$$
\lambda_{m f p}^{e}=\frac{1}{n_{n} \sigma_{e-n}}=7 \times 10^{-2} \mathrm{~m} .
$$

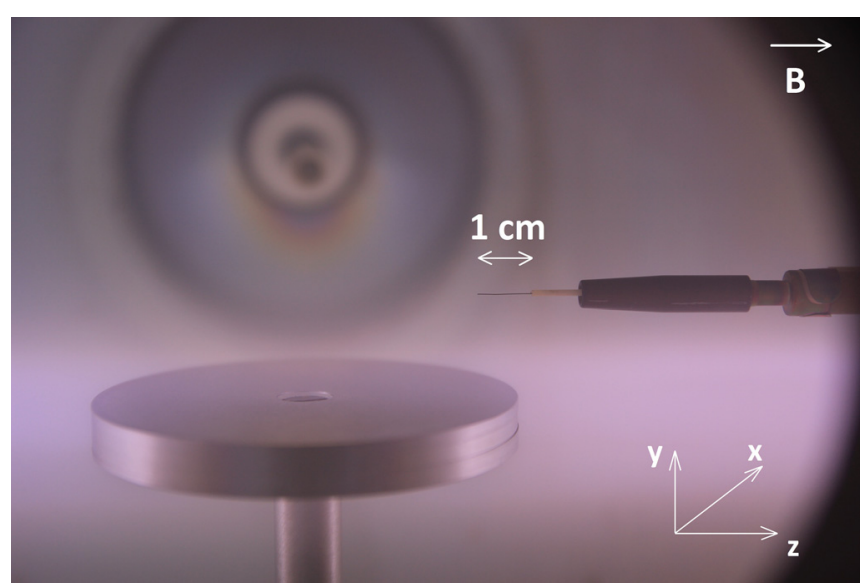

FIG. 1. Aline RF antenna and probe on the manipulator. 
Particles in the plasma can be considered as magnetized when their cyclotron frequencies exceed the collision frequency or, equivalently, their Larmor radii must be smaller than the mean free paths $r_{c \alpha}<\lambda_{m f p}^{\alpha}$

$$
r_{c \alpha}=\frac{\sqrt{m_{\alpha} k T_{\alpha}}}{q_{\alpha} B} .
$$

The magnetic field in Aline is varied up to $0.104 \mathrm{~T}$. It is beneficial to find a limit of the magnetic field below which electrons are not magnetized.

The minimum used field is $0.0024 \mathrm{~T}$. For this value, the condition of electron magnetization is fulfilled

$$
r_{c e}(0.0024 \mathrm{~T})=0.2 \times 10^{-2} \mathrm{~m}<7 \times 10^{-2} \mathrm{~m}=\lambda_{m f p}^{e} .
$$

Therefore, data processing for the whole working range of magnetic field values [0.0024; 0.104$] \mathrm{T}$ can be addressed with approximation of the collisionless magnetized plasma. Pressure diminution below the one considered here would make the mean free path bigger, and so, the conditions (3)-(4) would remain valid.

\section{B. Probe design for the magnetized plasma}

The probe design for the magnetic environment must be addressed with special care. A gap between the probe tip and the compensation electrode was present for one of our probes, bigger in size than the tip radius. It led to increased current collection for big magnetic fields since the mean electron Larmor radius is then comparable to the probe tip size, and so, a large fraction of electrons could penetrate inside the gap, and thus, the collecting area increased. In our experiments with unmagnetized plasmas, this effect was never observed, but in the presence of a magnetic field, it is important to not allow such gaps.

\section{CURRENT COLLECTION AT $\mathrm{V}_{\mathrm{pl}}$}

The equation for the electron current at $\mathrm{V}_{\mathrm{pl}}$ in the nonmagnetized plasma is well known, and it reads

$$
I_{e}=e \Gamma_{e} S_{p r}=e \frac{n \bar{v}}{4} S_{p r}=e n\left(\frac{k T_{e}}{2 \pi m_{e}}\right)^{1 / 2} S_{p r} .
$$

Electron flux to a surface in one direction $\Gamma_{e}$ $=n\left(\frac{k T_{e}}{2 \pi m_{e}}\right)^{1 / 2}$ is given through the mean velocity $\bar{v}$ of a Maxwellian distribution with no limitations neither on the value nor on the direction of the electron velocity. The electrons are collected by the whole probe surface $S_{p r}$.

Is it possible to employ this formula for magnetized plasmas? As was shown by some experiments, ${ }^{4}$ it is indeed applicable for certain conditions. A convenient parameter for numerical characterization is the dimensionless magnetic field strength $\beta$

$$
\beta=\frac{r_{p r}}{r_{c e}}=\frac{r_{p r} e B}{\sqrt{m_{e} k T_{e}}} .
$$

So, we should not speak about the magnetic field itself, rather about the ratio of the probe radius $r_{p r}$ and the electron
Larmor radius. In Ref. $4, \beta$ was in the range of $0.25-2$, and the resulting error of the electron density evaluation with (4) was estimated as $\pm 20 \%$ for a cylindrical probe perpendicular to the magnetic field.

However, not the $\beta$ parameter but the perpendicularity of the probe played the crucial role in that work, as our theory will demonstrate below. The collecting area of the probe in (4) was replaced by Kudrna et al. by 2 times the cross section of the probe in the plane perpendicular to the magnetic field. It is easy to see that the difference between this area and the whole surface of the probe is negligible for such a probe orientation. That is why the conventional theory was able to provide such good results.

The question that arises next is whether and how the theory should be corrected for a probe at an arbitrary angle to the magnetic field. We have obtained an idea of how to answer this question from an experiment.

A reference planar probe is used for comparison. All probes were placed at the same position inside the plasma chamber, and IV curves were captured at the same values of pressure and magnetic field. With the density from the planar probe, we are able, using the same equation (4), to get an idea of how the collecting area of a cylindrical probe looks like: $S_{c y l}=\frac{I_{c y l}}{I_{\text {planar }}} S_{\text {planar }}$. The result for the smaller cylindrical probe $\left(\mathrm{r}_{\mathrm{pr}}=75 \mu \mathrm{m}\right)$ is shown in Fig. 2.

We see that the same idea as in Ref. 4 would not work: the perpendicular area is $S_{\perp}=1.8 \times 10^{-8} \mathrm{~m}^{2}$ in our case, which is few orders of magnitude smaller than the experimental result. We clearly observe from the experiment that the collecting area of a cylindrical probe at a small angle to the magnetic field is not a constant but a function of the magnetic field strength.

\section{A. Effective collecting area}

Our theory suggests an alternative "effective" collecting area of a cylindrical probe at an arbitrary angle $\theta$ to the magnetic field. In (4), the electron current is calculated with the assumption of all particles having the same mean velocity.

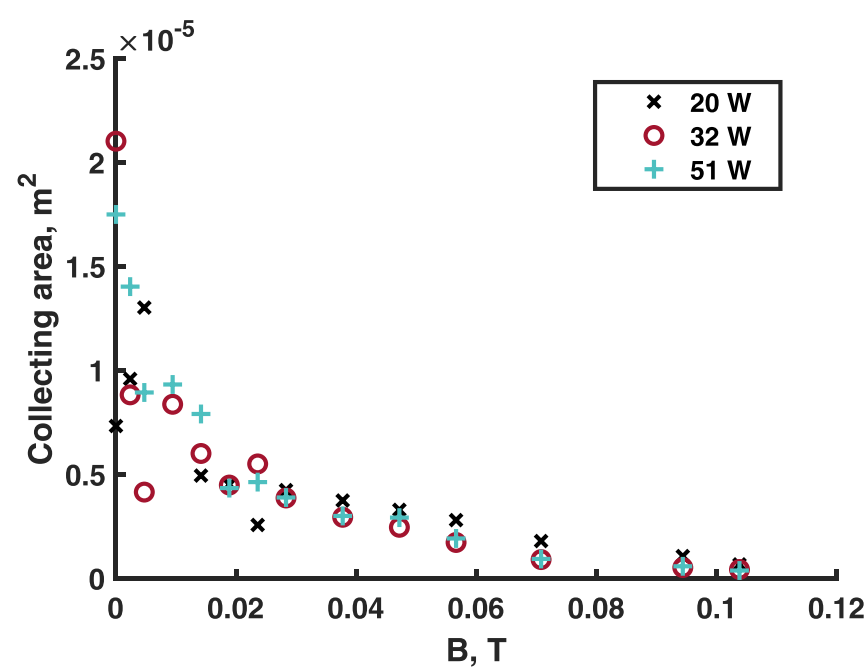

FIG. 2. Collecting area of the smaller cylindrical probe estimated using planar probe density measurements for 3 different forwarded RF power levels. 
In reality, electron velocities follow the Maxwellian distribution (which is also a subject of discussion but outside of the scope of this work). We will now recalculate the collected current $I_{e}=e \Gamma_{e} S$, accounting for different mechanisms of electron collection depending on their radial speed. We assume no dependency on longitudinal velocities.

We emphasize again that we only consider the probe potential equal to $\mathrm{V}_{\mathrm{pl}}$. We want to give a simple formula for density evaluation from this exact point of an IV curve, without diving into complex particle transport theories for the current collection by a biased probe.

Generally speaking, at any given magnetic field, the electrons have Larmor radii in some range of values. We assume that those with $r_{c e} \leq r_{p r}$ are collected by the probe perpendicular cross section

$$
S_{\perp}=2 r_{p r} L_{p r} \sin (\theta)+\pi r_{p r}^{2} \cos (\theta) .
$$

The mechanism of collection for the electrons with $r_{c e}$ $>r_{p r}$ is in principle the same as the collection of particles in the unmagnetized plasma. They are collected by the whole probe surface

$$
S_{p r}=2 \pi r_{p r} L_{p r}+\pi r_{p r}^{2}
$$

The electron current is then given by a nearly identical to (4) formula

$$
I=e \Gamma_{e} S_{e f f},=e n\left(\frac{k T_{e}}{2 \pi m}\right)^{1 / 2} S_{e f f},
$$

except that the collecting surface here is the effective $S_{\text {eff }}$. The current is calculated as a sum of two contributions

$$
I=e\left(\Gamma_{m a g n} S_{\perp}+\Gamma_{n-m a g n} S_{p r}\right) .
$$

Here, $\Gamma_{\text {magn }}$ is the flux to a surface, calculated for "magnetized" electrons which have $r_{c e} \leq r_{p r}$ or, equivalently, the velocity in the perpendicular to the $\mathrm{B}$ direction

$$
v_{r} \leq v_{\text {lim }}=\frac{e B r_{p r}}{m} .
$$

The non-magnetized flux $\Gamma_{n-m a g n}$, on the contrary, is calculated with the opposite condition on the electron velocity.

Since in fact we measure only net current in the experiment, not the two contribution of electrons separately, we do not want to speak about different currents but rather introduce the "effective collecting area" into terminology, defining it as

$$
S_{e f f}=I / e \Gamma_{e}=\left(S_{\perp} \Gamma_{m a g n}+S_{p r} \Gamma_{n-m a g n}\right) / \Gamma_{e} .
$$

A random particle flux to a surface is generally calculated as

$$
\Gamma=n \int \overrightarrow{v_{s}} f(v) d^{3} v
$$

with $\overrightarrow{v_{\mathrm{S}}}$ being a speed normal to the surface.
Without loss of generality, we can calculate a flux of magnetized electrons to a plane perpendicular to B in cylindrical coordinates (the $\mathrm{z}$-direction is parallel to B) and with Maxwellian velocity distribution

$$
\Gamma=n\left(\frac{m_{e}}{2 \pi k T_{e}}\right)^{\frac{3}{2}} * \int_{0}^{+\infty} \int_{0}^{2 \pi} \int_{0}^{+\infty} v_{z} v_{r} \exp \left(-\frac{m v^{2}}{2 k T_{e}}\right) d v_{r} d \varphi d v_{z}
$$

After integration for $v_{z}$ and $\varphi$

$$
\Gamma=n\left(\frac{m_{e}}{2 \pi k T_{e}}\right)^{\frac{1}{2}} \int_{0}^{+\infty} v_{r} \exp \left(-\frac{m v_{r}^{2}}{2 k T_{e}}\right) d v_{r}
$$

Applying limitation (10) instead of $(0,+\infty)$ in (14), we calculate the flux of the "magnetized" electrons

$$
\Gamma_{\text {magn }}=\Gamma_{e}\left(1-e^{-\frac{\beta^{2}}{2}}\right) .
$$

Similarly

$$
\Gamma_{n-\operatorname{magn}}=\Gamma_{e} e^{-\frac{\beta^{2}}{2}},
$$

with $v_{r}$ limited from $v_{\text {lim }}$ to $+\infty$.

Hence, we obtain the expression for the effective area

$$
S_{\text {eff }}=S_{\perp}\left(1-e^{-\frac{\beta^{2}}{2}}\right)+S_{p r} e^{-\frac{\beta^{2}}{2}} .
$$

For the unmagnetized plasma $(\beta=0)$, this expression turns into $S_{\text {eff }}=S_{p r}$, thus transforming (8) into conventional equation (4).

To give an idea of a typical profile of the effective collecting area given by (17), we provide two illustrations. In Fig. 3, we plot an example of the effective area for cylindrical probes of $1 \mathrm{~cm}$ length and 4 different radii, all parallel to the magnetic field. The electron temperature is kept constant at $\mathrm{T}_{\mathrm{e}}=3 \mathrm{eV}$. For the smallest radius, the value of $\beta$ is small for the whole range of $\mathrm{B}$, and so, the effective area declines very slowly. As the probe radius gets bigger, the curve

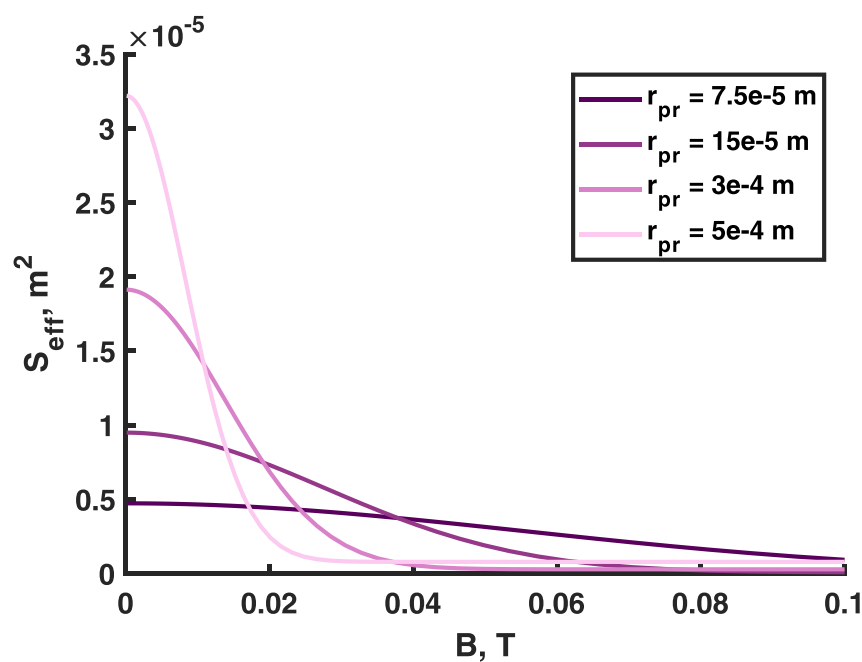

FIG. 3. Example of the effective collecting area for different probe radii at $\theta=0$. 
correspondingly bends more. The dependence on the angle is shown in Fig. 4, for the probe radius of $7.5 \times 10^{-5} \mathrm{~m}$. It is clear that the perpendicular projection of a cylindrical probe is the biggest when the probe is inclined at $90^{\circ}$ to the magnetic field. This contribution mostly plays a role at larger magnetic fields.

\section{B. Application to experimental data}

Now, we can check how good the developed theory works in practice. We calculate the effective collecting area for two cylindrical probes at an angle of $5^{\circ}$ to the direction of B using experimentally obtained data (realistic temperatures, varying for different points) and compare it to the collecting area estimated from planar probe density measurements as was described above and displayed in Fig. 2.

The agreement of the curves is remarkably good for bigger magnetic fields (Figs. 5 and 6). For the small fields, we believe the effective area to be the correct one, not the planar probe reference values, simply because the upper possible limit for the collecting area is the whole probe area (4.7 $\times 10^{-6} \mathrm{~m}^{2}$ and $3.2 \times 10^{-5} \mathrm{~m}^{2}$, respectively). The reason for the discrepancies with the collecting area from the planar probe at smaller B is the inaccurate estimation of the density for the planar probe in this region. Big uncertainty exists in the plasma potential evaluation from the IV curves at small magnetic fields due to the absence of a clear transition from the exponential to the electron saturation part, which leads to erroneous values of the current at $\mathrm{V}_{\mathrm{pl}}$ and calculated density (since the density is linearly proportional to the current).

Densities calculated using data from the planar probe and the cylindrical probes are compared in Fig. 7. The agreement of the results for all probes is satisfactory, considering typical probe measurement errors.

\section{Comparison to Laframboise-Rubinstein theory}

Theoretical papers of Laframboise and Rubinstein ${ }^{2,3}$ focus on cylindrical probes in the magnetized plasma. Their work covers all crucial parameters: the magnetic field

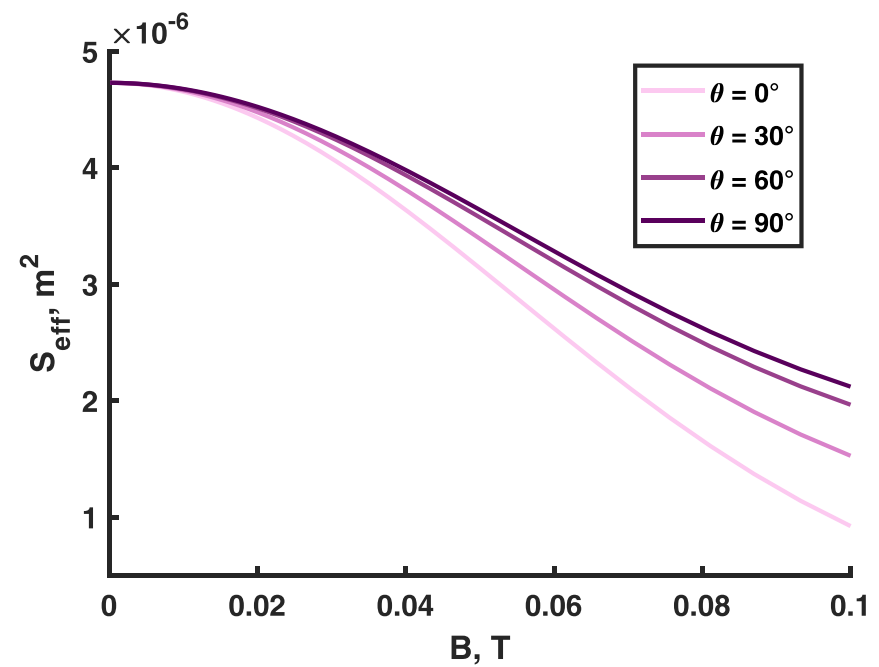

FIG. 4. Example of the effective collecting area for different probe inclination angles with $\mathrm{r}_{\mathrm{pr}}=75 \mu \mathrm{m}$.

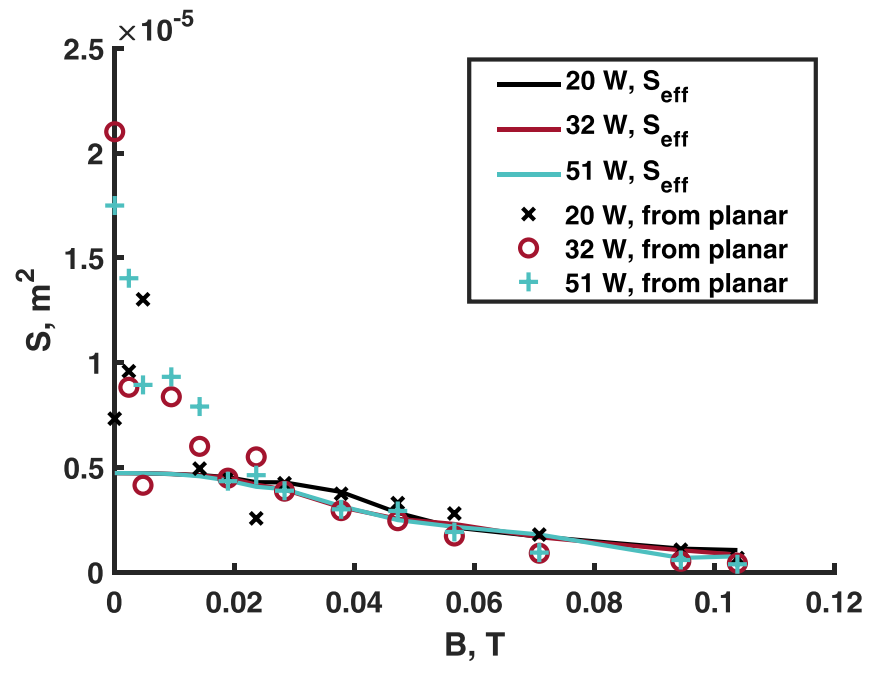

FIG. 5. Comparison of $\mathrm{S}_{\text {eff }}$ to the collecting area estimated using the planar probe for the smaller cylindrical probe.

strength and the probe size and orientation, providing upper bounds and adiabatic limits for the current collected by the probe at different biasing potentials. The authors claim that at $\mathrm{V}_{\mathrm{pl}}$, the upper bound and the adiabatic limit formulas coincide and give exact values of the current.

In Ref. 2, the current at $\mathrm{V}_{\mathrm{pl}}$ can be numerically calculated from the general integral for each possible $\theta$ and $\beta$. A plot is presented in Fig. 3 in Ref. 2 for the normalized current at $\mathrm{V}_{\mathrm{pl}}$.

We can construct a similar plot using our $\mathrm{S}_{\text {eff }}$ (Fig. 8). The current at $\mathrm{V}_{\mathrm{pl}}(8)$ is normalized to the current for $\beta=0$ : $\mathrm{I}_{0}=\mathrm{en}\left(\frac{\mathrm{kT}}{2 \pi \mathrm{m}}\right)^{1 / 2} \mathrm{~S}_{\mathrm{pr}}$, so that $\mathrm{i}=\mathrm{I} / \mathrm{I}_{0}$. In Ref. 2, the probe length is assumed infinite. We make calculations for our probe with dimensions $\mathrm{L}_{\mathrm{pr}}=1 \mathrm{~cm}$ and $\mathrm{r}_{\mathrm{pr}}=75 \mu \mathrm{m}$, so that $\frac{\mathrm{L}_{\mathrm{pr}}}{\mathrm{r}_{\mathrm{pr}}} \gg 1$, and it can also be assumed infinite.

The currents agree well for big inclination angles and big dimensionless magnetic fields. The cause of the discrepancies at small $\theta$ is that the current in Ref. 2 is calculated only for the electron collection on the side of the probe. The

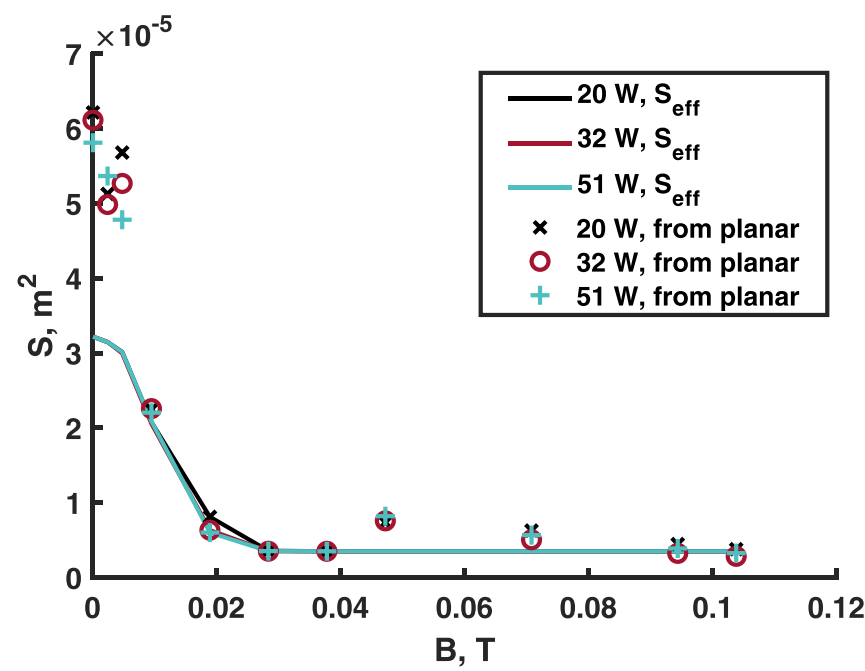

FIG. 6. Comparison of $\mathrm{S}_{\text {eff }}$ to the collecting area estimated using the planar probe for the bigger cylindrical probe. 


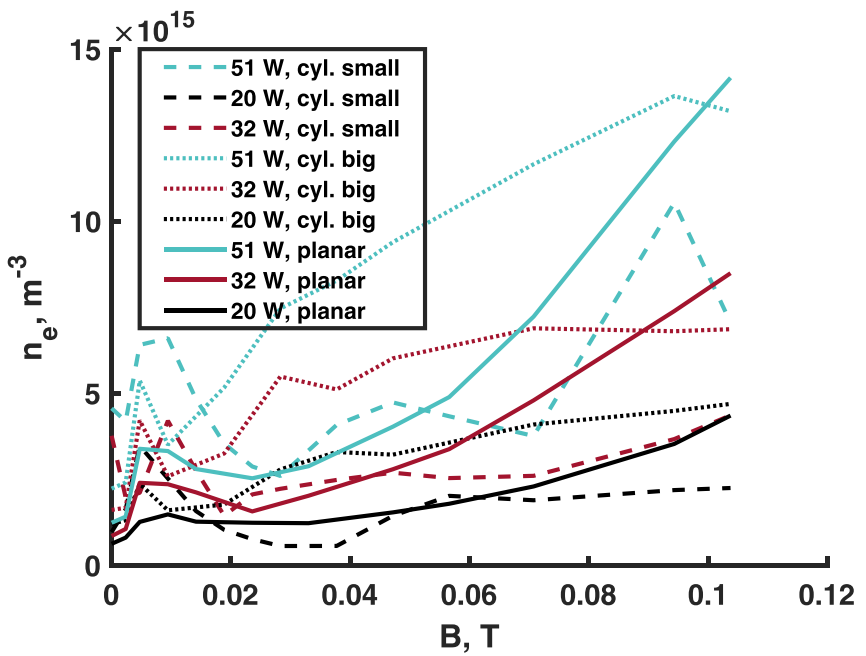

FIG. 7. Comparison of densities calculated for two cylindrical probes at $5^{\circ}$ and the planar probe.

end-effect current is given separately in Ref. 2 by the following expression (here, $\sigma=\beta * \pi^{1 / 2} / 2$ ):

$$
\frac{I}{I_{0}^{*}}=\frac{1}{2}\left(1+\frac{1-\exp \left(-\sigma^{2}\right)}{\sigma^{2}}+\frac{\pi^{\frac{1}{2}}(1+\operatorname{erfc}(\sigma))}{\sigma}\right) .
$$

It is not trivial to combine this expression for the endeffect current to the previous part. For the end-effect current, $I_{0}^{*}$ is the current received by the perpendicular area of the probe (much smaller than the parallel area), and for a semiinfinite probe in the Laframboise-Rubinstein model, it leads to a current rise in (18) up to infinity for $\beta \rightarrow 0$.

Our theory, on contrary, does not have any discontinuities in solutions and smoothly converges in the extrema to two possible solutions: unmagnetized case $\mathrm{i}(\theta)=1$ for $\beta \rightarrow 0$ (for any angle) and to $\mathrm{i}=\frac{\mathrm{S}_{\perp}}{\mathrm{S}_{\mathrm{pr}}}$ for $\beta \rightarrow \infty$ and relatively small $\theta$. For $\beta \rightarrow \infty$ and not very small $\theta$, the parts proportional to $r_{p r}^{2}$ in (6) and (7) are insignificant, and so, $\mathrm{i} \rightarrow \frac{2 \sin \theta}{\pi}$. The interesting fact is that the same dependence formula $\mathrm{i}=\frac{2 \sin \theta}{\pi}$ is mentioned in Ref. 3, which they derived using a different approach.

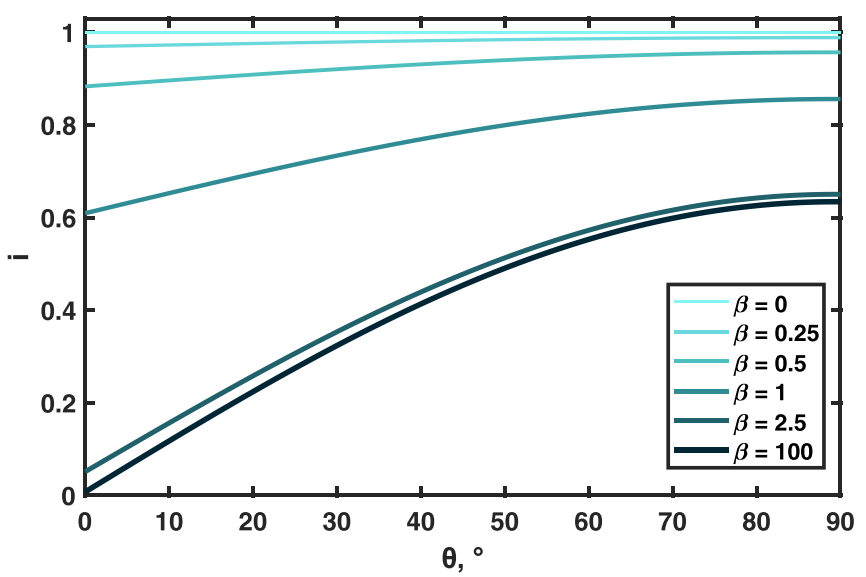

FIG. 8. Normalized current $\mathrm{i}$ as a function of the probe inclination angle for various dimensionless magnetic fields.

\section{Peculiarities of the theory application}

Two important aspects must be mentioned here. One concerns the fact that the probe perpendicular surface can be taken either once or twice in calculations. The other remark is about the assumption of an infinite probe and about probes with various finite dimensions.

In the processing of our experimental data, the probe perpendicular area $S_{\perp}$ was taken as in (6). Behind our probe, there is a holder with a ceramic insulator, and so, the electrons can only be collected from one side. It is also taken into account in (7) for the whole probe area.

On the contrary, Fig. 8 was made for a probe that can collect particles from both directions along the magnetic field lines (but with the same area $S_{\mathrm{pr}}$ ). It is closer to the conditions in which the theory in Ref. 2 was constructed. That is why a surface double as (6) was taken. To give an idea of how the current collection would change, we plot the same currents as in Fig. 8 but take a single perpendicular area instead of a double (Fig. 9). The difference is especially significant for big $\beta$ and $\theta$, and so, the correct perpendicular area must be accurately chosen for each specific case.

The second valuable aspect is the case of a finite probe. We provide an example of how the current collection would change for varying probe dimensions (Fig. 10).

As would be empirically expected, the line for a probe with the length equal to the diameter $L_{p r}=2 r_{p r}$ is nearly symmetric. Such a probe would be close to a cube, and so, the perpendicular and planar orientations are nearly equal in terms of the collected current. As the ratio $\frac{L_{p r}}{2 r_{p r}}$ grows, the collected current approaches the extremum of the infinite probe. The last value is given for the dimensions of our cylindrical probe, the same as it was used for Fig. 8. All lines are for the same $\beta=2$.

An important conclusion that can be drawn here is that our theory is not limited to the cylindrical shape of the probes. It can be applied to any shape, as long as the whole surface and its perpendicular projection are known.

\section{PLASMA POTENTIAL}

The method of density evaluation at the plasma potential with the effective collecting area is useless without a

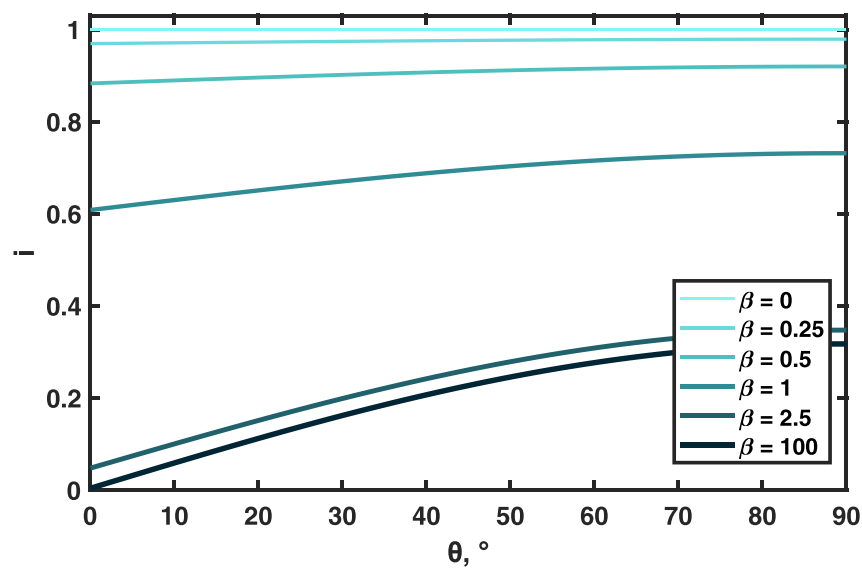

FIG. 9. $\mathrm{i}(\theta)$ for various $\beta$. The perpendicular current collection is only from one direction. 


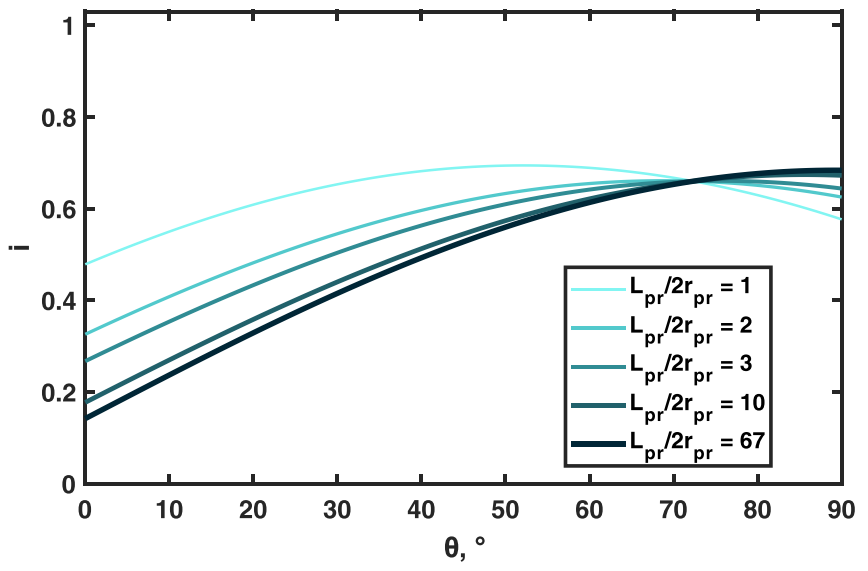

FIG. 10. $i(\theta)$ for various $\frac{L_{p r}}{2 r_{p r}}($ at $\beta=2)$.

sufficiently precise and robust technique for evaluation of the plasma potential itself. In this section, we examine the conventional method of $\mathrm{V}_{\mathrm{pl}}$ calculation from the maximum of the first derivative of the current and introduce another method, which showed better robustness in our applications.

\section{A. Plasma potential from the 1st derivative}

The most common method of $\mathrm{V}_{\mathrm{pl}}$ calculation from the first derivative of the current often gives too low unrealistic values. Taking the first minimum of the second derivative after the point where the first derivative has maximum is another possible method. It gives slightly bigger values for $\mathrm{V}_{\mathrm{pl}}$ but is only applicable to data with a very low noise level.

The underestimation of $\mathrm{V}_{\mathrm{pl}}$ values is not the only weakness of the first derivative approach. More importantly, a reason for the search of alternative methods is given by the revealed low robustness of the first derivative technique. It is illustrated in the following example.

The IV characteristics (smaller cylindrical probe) for similar values of 0.0236 and $0.0283 \mathrm{~T}$ are nearly the same for each RF power (Fig. 11). Consequently, $\mathrm{V}_{\mathrm{pl}}$ and the current at $\mathrm{V}_{\mathrm{pl}}$ should be nearly the same. It is not true for the $32 \mathrm{~W}$ power level. A bump which is present at the bigger

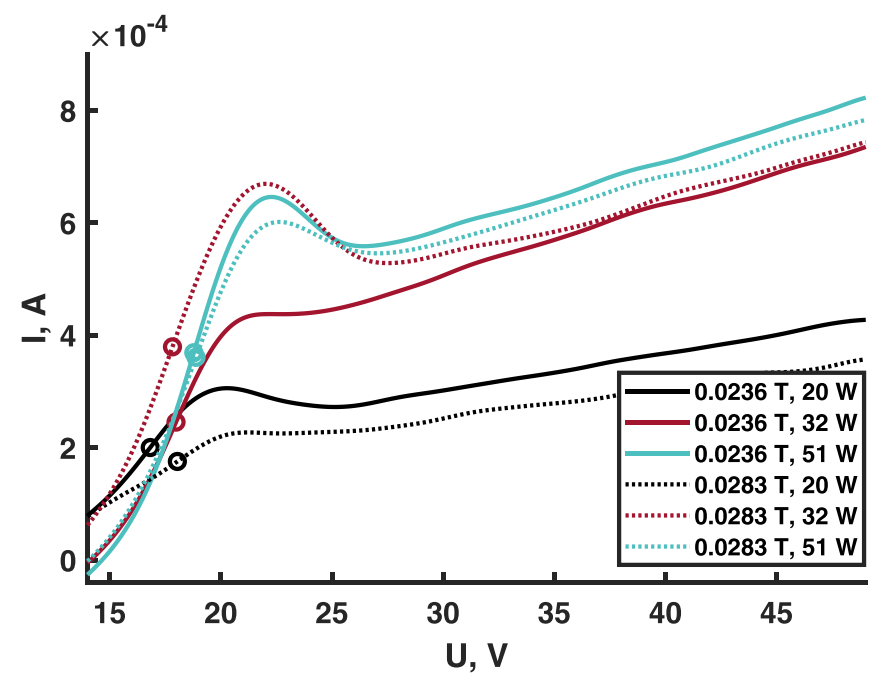

FIG. 11. Example of $\mathrm{V}_{\mathrm{pl}}$ from the 1st derivative method (circle markers).
$0.0283 \mathrm{~T}$ field does not appear at $0.0236 \mathrm{~T}$. The nature of this bump is not fully understood. An attempt to study this phenomenon has been done in Refs. 5 and 6, showing that there is a dependence of the bump height on the magnetic field strength and on the ratio of the probe radius and length. This bump changes the shape of the first derivative, thus influencing the obtained $\mathrm{V}_{\mathrm{pl}}$. This causes a difference of two times in the current at $\mathrm{V}_{\mathrm{pl}}$ and hence the same difference in the density evaluated using this current.

One more type of a problem has been observed when using the 1 st derivative technique. The sought peak of $\mathrm{dI} / \mathrm{dV}$ is not always clear. If it is blurred, an uncertainty in $\mathrm{V}_{\mathrm{pl}}$ identification of the order of few volts can follow. It has been noted from our experimental data that a shift of $1 \mathrm{~V}$ in $\mathrm{V}_{\mathrm{pl}}$ can cause a difference of more than $100 \%$ in the current at $\mathrm{V}_{\mathrm{pl}}$, and consequently, it affects the electron density. This effect is especially strong for the IV curves obtained in the presence of a high magnetic field. The transition region is very narrow in this case, only few volts.

\section{B. Plasma potential at intersection}

In this method, $\mathrm{V}_{\mathrm{pl}}$ is found as the $\mathrm{x}$-coordinate of the intersection of 2 lines:

- linear interpolation of the region between $\mathrm{V}_{\mathrm{fl}}$ and $\mathrm{V}_{\mathrm{pl}}$ and

- linear interpolation of the electron saturation current.

Not the full regions are taken but only those parts which have a linear shape. For our data, it was the last $30 \mathrm{~V}$ of the electron saturation current and $1 / 3$ of the region between $V_{\mathrm{fl}}$ and the first approximation of $\mathrm{V}_{\mathrm{pl}}$ found with the classical 1st derivative approach.

A similar technique has been published (for example, Ref. 14). The difference is that usually the current is taken on a log scale. In our data, there is no clear linear region for the transition part of the IV curve on the log scale. That is why the "log intersection" method is not applicable, but the intersection at the linear scale works, as will be shown below.

The same data as in Sec. II A are used to compare the techniques. In Fig. 12, the prolonged lines of the fits and the obtained points of the intersection can be seen. It can be seen that this method is not affected by the bump phenomenon. With this technique, $\mathrm{V}_{\mathrm{pl}}$ for $32 \mathrm{~W}$ and the corresponding values of the current are similar for the two magnetic field values. The tendency of the increase in the current at $\mathrm{V}_{\mathrm{pl}}$ with the increase in the injected RF power is also a good sign.

The intersection method is not always applicable. For very small magnetic fields $(<0.005 \mathrm{~T})$, the knee of an IV curve is hard to define since the slopes of the two adjoining regions are very similar. In such cases, it is impossible to apply the intersection method. $\mathrm{V}_{\mathrm{pl}}$ from the 1 st derivative is taken instead.

The evaluation of the plasma potential with the intersection technique results in a point right at the edge of the knee. This plasma potential estimation is reliable in low-pressure, unmagnetized, dc discharges. In collisional or magnetized plasmas, the magnitude of $\mathrm{I}_{\mathrm{es}}$ is supposed to be lowered and the knee blurred, and so, the real $\mathrm{V}_{\mathrm{pl}}$ becomes hard to 


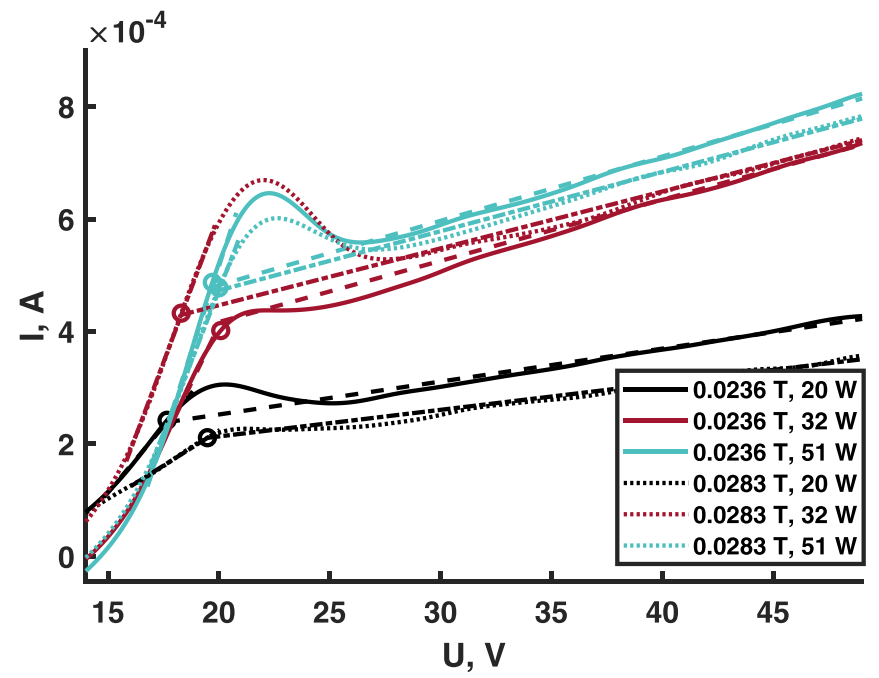

FIG. 12. Example of $\mathrm{V}_{\mathrm{pl}}$ at intersection (circle markers).

determine. In addition, $\mathrm{RF}$ fluctuations of $\mathrm{V}_{\mathrm{pl}}-\mathrm{V}_{\mathrm{pr}}$ can be significant when the RF amplitude is much bigger than $\mathrm{kT}_{\mathrm{e}} / \mathrm{e}$.

If $\mathrm{V}_{\mathrm{pl}}$ and the current at $\mathrm{V}_{\mathrm{pl}}$ are overestimated, it can lead to

(1) larger density values due to linear dependency $n \sim I$ and

(2) imprecise collecting area definition. At values higher than the real plasma potential, an expansion of a sheath begins, and so, a bigger area must be used in calculations. Since $n \sim 1 / A$, it adds up to the rise of the resulting density estimation.

Error bars for the density can be obtained for each case by comparing the current at $\mathrm{V}_{\mathrm{pl}}$ for the two techniques. In our experiments, this ratio was typically in the range of 1-2. Possible sheath expansion was neglected.

The important fact is that the robustness of the intersection approach is much higher than the 1st derivative one. More consistency of the behavior of the current at $\mathrm{V}_{\mathrm{pl}}$ can be seen in Fig. 13. This fact outweighs possible consequences of slight overestimation of $\mathrm{V}_{\mathrm{pl}}$ and current at $\mathrm{V}_{\mathrm{pl}}$ values.

In the end, we conclude that processing Langmuir probe data in the presence of a magnetic field using only the 1st

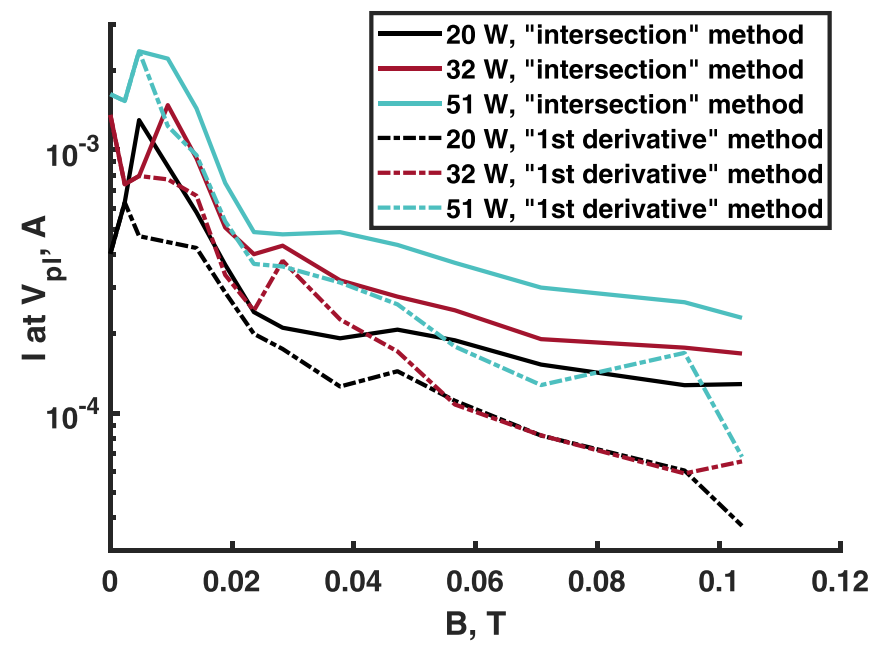

FIG. 13. Comparison of the current at $\mathrm{V}_{\mathrm{pl}}$ for two methods. derivative method is not acceptable. The intersection technique is adopted as more reliable, with an exception for low $B$ values, leading to the final combined method.

\section{TEMPERATURE}

In Langmuir probe data processing, the precision of the estimation of all relevant parameters is important since they are all self-consistent and an error in one parameter enhances errors in others. Here, we describe our approach for the calculation of the electron temperature.

The electron temperature can be obtained from the slope of an IV curve between floating and plasma potentials. The electron current as a function of voltage for $\mathrm{V} \leq \mathrm{V}_{\mathrm{pl}}$ is expressed as ${ }^{15}$

$$
I_{e}(V)=I_{e s} \exp \left(-\frac{e\left(V_{p l}-V\right)}{k T_{e}}\right) .
$$

A slope of a linear fit to the logarithm of electron current is used to determine $\mathrm{Te}$

$$
T_{e}=\frac{V_{2}-V_{1}}{\ln I_{2}-\ln I_{1}},
$$

where indexes 1 and 2 refer to any two points on the line.

An inverse mean value of the gradient $\frac{d \ln I_{2}}{d V}$ for a part of the region between $\mathrm{V}_{\mathrm{fl}}$ and $\mathrm{V}_{\mathrm{pl}}$ is taken in our data processing. This part is the central third part of the whole span. The reason why we do not take the whole region is that the measured current is a sum of the electron and the ion current, which means that the pure exponential shape is disturbed. The measured current is shifted down from the values of the electron current by some $\mathrm{I}_{\mathrm{i}}(\mathrm{V})$. The ion current in this region is not constant, and it decays towards $\mathrm{V}_{\mathrm{pl}}$, but since it happens relatively slowly and the determination of the correct law for this decay is very sophisticated in the presence of the magnetic field, we assume it to be constant in our calculations.

A shift of an exponential function by a constant does not result in a parallel linear shift of the same function on the log scale (Fig. 14). It means that if the whole region from $V_{\text {fl }}$ to $\mathrm{V}_{\mathrm{pl}}$ is considered, the current would look like the brightest line $(y=\exp (x)-1)$ in Fig. 14.

That is why the whole region should not be used for the temperature evaluation. The resulting average curve slope will be overestimated, and consequently, the temperature will be underestimated. When the used region is cut on the left at around $1 / 3$, the explained error can be strongly reduced. The reason to cut $1 / 3$ on the right has a different nature: $\mathrm{V}_{\mathrm{pl}}$ can be overestimated as was previously discussed, and so, we ignore the part close to $\mathrm{V}_{\mathrm{pl}}$.

We regard the temperatures obtained from the central part of the current linear fit on a $\log$ scale as the lower limit. Then, we use it as the starting point for an iterative algorithm that self-consistently calculates densities and temperatures. First, the temperature is used to determine the density, and then, the ion current is calculated theoretically for a point of the voltage that is approximately the value of $\mathrm{T}_{e}$ (in $\mathrm{eV}$ ) below $\mathrm{V}_{\mathrm{pl}}$ 


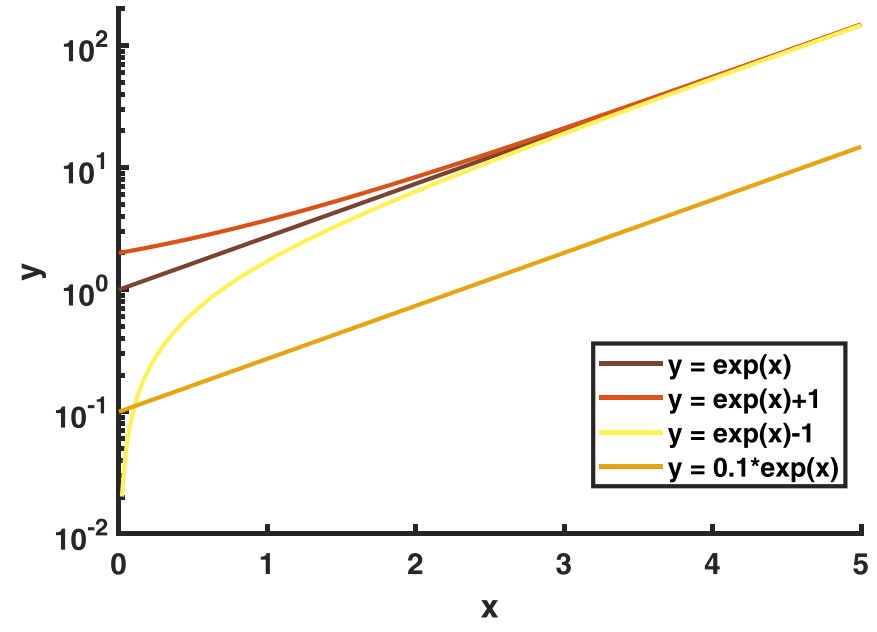

FIG. 14. Shifted exponential functions on a logarithmic scale.

$$
I_{i}=0.6 e n\left(\frac{k T_{e}}{2 \pi m_{i}}\right)^{1 / 2} S_{i}
$$

The equation for the collecting area $S_{i}$ is the same as for the whole probe surface (7) except that instead of the probe radius, the sum of the radius and the sheath length $\mathrm{s}$ is taken. At that point, the negative sheath is not yet too large and can be determined as given in Ref. 16

$$
s(V)=\frac{2}{3}\left(\frac{\varepsilon_{0}}{I_{i} / S_{i}}\right)^{1 / 2}\left(\frac{2 e}{m_{i}}\right)^{1 / 4}\left(V_{p l}-V\right)^{3 / 4} .
$$

Taking the ratio $I_{i} / S_{i}$ from (20) and the Debye length definition $\lambda_{D}=\left(\frac{\varepsilon_{0} k T}{n e^{2}}\right)^{1 / 2}$, Eq. (21) transforms into

$$
s(V)=1.02 \lambda_{D}\left(\frac{e\left(V_{p l}-V\right)}{k T_{e}}\right)^{3 / 4} .
$$

The calculated ion current is then subtracted from the net current, and the result is used to determine the next iteration of $T_{e}$, the same way as was described above, from the central part of the current between $\mathrm{V}_{\mathrm{fl}}$ and $\mathrm{V}_{\mathrm{pl}}$ on a $\log$ scale. The iterations continue until a stable value of the temperature is reached.

The described procedure was applied to the data processing of the cylindrical probe. For the planar probe, the ion saturation current is negligibly low compared to the electron saturation current, and so, the iterative algorithm does not change much the values of the lower limit of the temperature. Resulting temperatures acquired with the planar probe are similar to the ones from the cylindrical probes and have the same tendency to descend with increasing $B$ and $n_{e}$ (Fig. 15).

The transition region used for the density evaluation is very narrow for big magnetic fields, and so, the increased error level may be expected there.

While the temperature obtained after the iterations is higher than the lower limit and closer to the real one, it is still underestimated. The proper procedure would be to subtract the ion current as a function of the voltage. Since it is a descending function (in absolute values), the right part of the

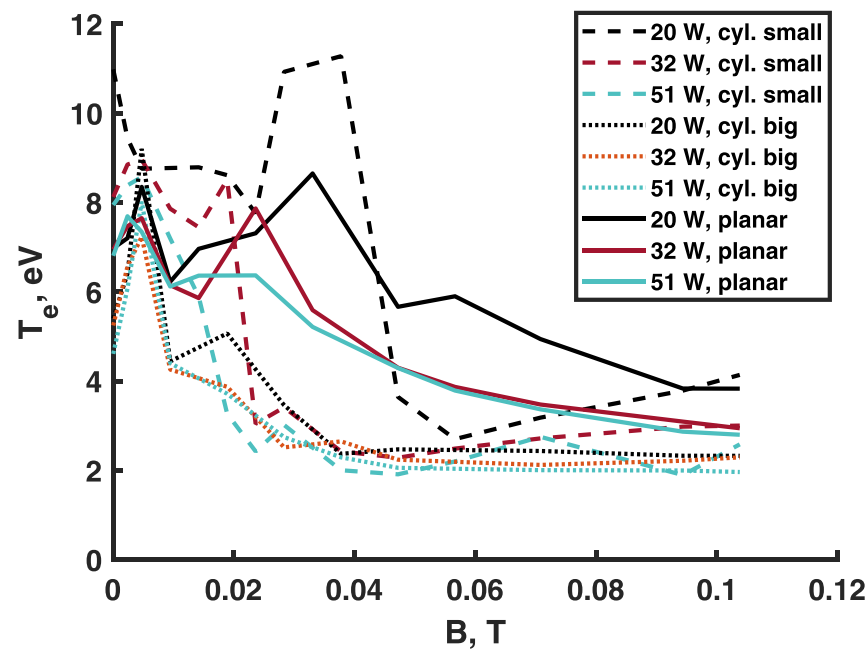

FIG. 15. Temperature comparison for three probes.

net current should be shifted less after the subtraction, which means that the slope will be lower and the temperature will be higher. We ignore this effect in our data processing, but it might be important to take it into account for some other conditions.

\section{CONCLUSION}

The explained theory of the effective collecting area calculation provides a rather simple method of density evaluation with a cylindrical Langmuir probe in magnetized plasmas. The experimental validation of the applicability has shown positive results, which allows further use of cylindrical probes in magnetized conditions at various angles. The advantage of such diagnostic is the accuracy of local measurements, allowing precise mapping of a small plasma region.

In the proximity of the extreme limit (1), the theory approaches the existing technique of using a perpendicular projection of a probe as the collecting area. On the other hand, the constructed formula demonstrates that for the increasing dimensionless magnetic field (with all other plasma parameters unchanged), the electron current will be reduced only to some limit and then will remain constant. This matches our experimental observations.

Correct plasma potential evaluation is crucial for further calculations of the density from the current at the plasma potential. The conventional technique employing the $1 \mathrm{st}$ derivative of the current is ineligible for the magnetized plasma due to the explained low robustness. The intersection method is a better alternative, shown to be consistent for various magnetic fields. The only exception appears when the field is very small because the transition and the electron saturation regions are indistinguishable. In those occasions, the 1 st derivative technique is employed as the only possible one.

A detailed explanation is given for the procedure of the electron temperature evaluation from a part of the transition region. The resulting values seem to be quite accurate, and this accuracy is extremely valuable for the correct density estimation. 
The presented theory for the effective collecting area can be applied to a broad range of experimental conditions. No limitation on the probe size nor orientation is imposed. The shape of the probe is also not limited to the cylinder only. As long as the probe whole surface and the perpendicular B projection area are known, the theory remains valid.

\section{ACKNOWLEDGMENTS}

This work was carried out within the framework of the EUROfusion Consortium and received funding from the Euratom research and training programme 2014-2018 under Grant Agreement No. 633053. The views and opinions expressed herein do not necessarily reflect those of the European Commission.

${ }^{1}$ M. Tichy, P. Kudrna, J. Behnke, C. Csambal, and S. Klagge, J. Phys. IV 7(C4), 397-411 (1997).

${ }^{2}$ J. G. Laframboise and J. Rubinstein, Phys. Fluids 19, 1900 (1976).

${ }^{3}$ J. Rubinstein and J. G. Laframboise, Phys. Fluids 21, 1655 (1978).
${ }^{4}$ P. Kudrna and E. Passoth, Contrib. Plasma Phys. 37, 417-429 (1997).

${ }^{5}$ I. Mihaila, L. Solomon, C. Costin, and G. Popa, Contrib. Plasma Phys. 53(1), 96-101 (2013).

${ }^{6}$ I. Mihaila, S. Costea, C. Costin, and G. Popa, Contrib. Plasma Phys. 54(3), 291-297 (2014).

${ }^{7}$ T. K. Popov, P. Ivanova, M. Dimitrova, J. Kovačič, T. Gyergyek, and M. Cerček, Plasma Sources Sci. Technol. 21, 025004 (2012).

${ }^{8}$ T. K. Popov, M. Dimitrova, P. Ivanova, J. Kovačič, T. Gyergyek, R. Dejarnac, J. Stöckel, M. A. Pedrosa, D. López-Bruna, and C. Hidalgo, Plasma Sources Sci. Technol. 25, 033001 (2016).

${ }^{9}$ E. Faudot, S. Devaux, J. Moritz, S. Heuraux, P. M. Cabrera, and F. Brochard, Rev. Sci. Instrum. 86, 063502 (2015).

${ }^{10}$ S. Devaux, E. Faudot, J. Moritz, and S. Heuraux, Nucl. Mater. Energy 12, 908-912 (2017).

${ }^{11}$ I. D. Sudit and F. F. Chen, Plasma Sources Sci. Technol. 3, 162 (1994).

${ }^{12}$ P. A. Chatterton, J. A. Rees, W. L. Wu, and K. Al-Assadi, Vacuum 42(7), 489-493 (1991).

${ }^{13} \mathrm{G}$. Franz, Low Pressure Plasmas and Microstructuring Technology (Springer, 2009), ISBN: 978-3-540-85849-2.

${ }^{14}$ R. L. Merlino, Am. J. Phys. 75, 1078 (2007).

${ }^{15}$ F. F. Chen, Lecture Notes on Langmuir Probe Diagnostics (Electrical Engineering Department, University of California, Los Angeles, 2003).

${ }^{16} \mathrm{P}$. Chabert, Physics of Radio-Frequency Plasmas (Cambridge University Press, 2011), ISBN 978-0-521-76300-4. 